\title{
Capsule Commentary on Lynch et al., Geographic and Racial/ Ethnic Variations in Patterns of Multimorbidity Burden in Patients with Type 2 Diabetes
}

\author{
Omolola Adepoju, PhD, MPH \\ Accountable Care and Health Homes Programs, United Health Group, Sugar Land, TX, USA.
}

J Gen Intern Med 30(1):93

DOI: $10.1007 / \mathrm{s} 11606-014-3005-8$

(c) Society of General Internal Medicine 2014

$\mathrm{R}$ ural populations lag behind suburban and urban residents in nearly all health status indicators. It is also well established that racial and ethnic minorities are disproportionately affected by the burden of chronic illnesses, including diabetes. The study by Lynch et al. ${ }^{1}$ frames the discourse around multimorbidity, examining patterns of medical and mental multimorbidity among persons with Type 2 diabetes. The authors retrospectively examined a national cohort of approximately 900,000 veterans with diabetes and defined multimorbidity by 1 ) the number of comorbidities and 2) the pattern of comorbidities (medical, mental, both). Compared to other race/ethnicity combinations, non-Hispanic blacks reported significantly higher counts of comorbidities and had a $25-49 \%$ greater risk of medical, mental, and combined multimorbidity. Rural veterans were also more likely to have higher counts of comorbidities and significantly greater medical, mental, and combined multimorbidity. The examination of both number and pattern of comorbidities is a major strength of this study.

Others have reported that the number of comorbidities alone may not accurately measure the burden of illness; hence, the development and validation of indices that assign weights to certain comorbid conditions. ${ }^{2-4}$ Along with these chronic disease indices are measures that assign flags for grouping comorbidities in a standardized way. ${ }^{5}$ The simple analysis by Lynch et al. presents an additional evidence-base for targeted interventions to rural and non-Hispanic black populations; however, future work should incorporate validated measures of disease burden, such as the Elixahuser comorbidity measure. Such validated measures provide more specifics regarding the groups of comorbidities and provide a clearer direction for addressing multimorbidity in primary care settings.
Reducing multimorbidity disparities might play a major role in reducing the healthcare disparities gap. While the Patient Protection and Affordable Care Act (PPACA) offers the promise of reducing disparities by promoting access to equitable and efficient healthcare, we see within the Department of Veteran Affairs (VA), where all veterans are presumed to have access to health care, that mere access alone does not result in equitable health care outcomes. Targeted interventions to populations that bear the greatest burden of disease should consider non-routine elements that are culturally sensitive and promote timely and continuous access to care.

Conflict of Interest: The author has no conflicts of interest with this article.

Corresponding Author: Omolola Adepoju, $\mathrm{PhD}, \mathrm{MPH}$; Baylor College of Medicine Section of Health Services Research, Veterans Affairs Health Services Research and Development, Center for Innovations in Quality, Effectiveness and Safety, Michael E. DeBakey Veterans Affairs Medical Center, Houston, TX, USA (e-mail: omololaelizabeth.adepoju@va.gov).

\section{REFERENCES}

1. Lynch CP, Gebregziabher M, Axon AN, Hunt KE, Payne E, Egede LE. Geographic and Racial/Ethnic Variations in Patterns of Multimorbidity Burden in Patients with Type 2 Diabetes. J Gen Intern Med. 2014; (Article SPI \#2990)

2. van Walraven C, Austin PC, Jennings A, Quan H, Forster AJ. A modification of the Elixhauser comorbidity measures into a point system for hospital death using administrative data. Med Care. 2009;47:626-633.

3. Charlson ME, Pompei P, Ales KL, et al. A new method of classifying prognostic comorbidity in longitudinal studies: development and validation. J Chron Dis. 1987;40:373-383.

4. Escobar GJ, Greene JD, Scheirer P, et al. Risk-adjusting hospital inpatient mortality using automated inpatient, outpatient, and laboratory databases. Med Care. 2008;46:232-239.

5. Elixhauser A, Steiner C, Harris DR, et al. Comorbidity measures for use with administrative data. Med Care. 1998;36:8-27.

Published online November 1, 2014 